LETTER

\title{
Initial Codebook Algorithm of Vector Quantizaton
}

\author{
ShanXue CHEN ${ }^{\dagger \dagger \dagger a)}$, FangWei $\mathrm{LI}^{\dagger \dagger}$, WeiLe $\mathrm{ZHU}^{\dagger}$, Nonmembers, and TianQi ZHANG ${ }^{\dagger \dagger}$, Member $^{\circ}$
}

\begin{abstract}
SUMMARY A simple and successful design of initial codebook of vector quantization (VQ) is presented. For existing initial codebook algorithms, such as random method, the initial codebook is strongly influenced by selection of initial codewords and difficult to match with the features of the training vectors. In the proposed method, training vectors are sorted according to the norm of training vectors. Then, the ordered vectors are partitioned into $N$ groups where $N$ is the size of codebook. The initial codewords are obtained from calculating the centroid of each group. This initializtion method has a robust performance and can be combined with the VQ algorithm to further improve the quality of codebook.

key words: initialization method, computational complexity, vector quantization, image processing
\end{abstract}

\section{Introduction}

Vector quantization (VQ), which has been successfully used in various applications involving VQ-based encoding and VQ-based recognition, is an efficient technique for data compression and retrieval due to its excellent rate-distortion performance. The generalized Lloyd algorithm (GLA) [1], also known as the k-means algorithm, is the most cited and widely used VQ method due to its simplicity and relatively good fidelity. However, the GLA being a greedy algorithm, its performance is sensitive to initialization. An improper initial selection of code vectors leads the codebook design algorithm to converge into the local minima closest to the initial point.

To avoid the suboptimal problem of GLA, Laha et al. [2] proposed a method using the self-organizing feature map algorithm and surface fitting. Sun et al. [3] proposed an improved method using a combined scheme of principal component analysis (PCA) and genetic algorithm (GA). Kim et al. [4] proposed an improved method using wavelet transform and enhanced SOM algorithm. These methods demonstrated performance better than the GLA. They all use initial codebook based on the random selection method so the considerable computation time and arithmetical operations are needed to get optimal code vectors.

An improved algorithm of the GLA was developed by Thangavel and Kumar [5]. By using standard deviation to

Manuscript received January 21, 2008.

Manuscript revised March 6, 2008.

${ }^{\dagger}$ The authors are with Electronic Engineering College, University of Electronic Science and Technology of China, Chengdu, 610054 , China.

${ }^{\dagger \dagger}$ The authors are with Communication and Information Engineering College, ChongQing University of Posts and Telecommunications, ChongQing, 400065, China.

a) E-mail: chee420@163.com

DOI: 10.1093/ietisy/e91-d.8.2189 construct the Voronoi cells of equal deviation, they design a VQ codebook algorithm. As a splitting algorithm, it suffers from a serious complexity barrier. To reduce the computational complexity of a full search in the GLA, a fast VQ algorithm based on nearest set search was proposed by Qian [6]. Qian's method cannot improve the quality of codebook. This Letter proposes a method to improve and speed up the GLA by using the training vector features to construct initial codebook.

\section{Proposed Method}

The splitting method is a good method for initial codebook design. For the splitting method of Linde et al. [1], the performance of initial codebook will be influenced by the use of the perturbation vector in each splitting step. So this method has some uncertainties. The method of Thangavel and Kumar [5] resolves the uncertain question based on the use of standard deviation of each partition cell. As the splitting method, they all suffer from a serious complexity barrier that greatly limits their practical use. The random algorithm is a simple and direct method for initial codebook design. For a codebook of size $N$ and training vectors of size $M$, the initial codewords can be obtained by choosing $N$ vectors from $M$ training vectors based on random selection or selecting a vector as a codeword every $p$ vectors where $p=M / N$. The method of equal interval selection is a better random method that can avoid the question of no code vectors in some cells. The random method may select some nontypical vectors as the codewords that influence the performance of codebook.

In the proposed method, the training vectors are sorted according to the norm of each vector components. The ordered vectors are partitioned into $N$ groups where each group has $p$ vectors. The initial codewords can be obtained from calculating the centroid of each group. For smooth images, two vectors (image blocks) that have the approximate norm are usually the similar vectors. For images with a large amount of detail, this situation usually can't be satisfied. The better initial codebook can be obtained by clustering the similar vectors. So compared to the random method, the proposed initial method presents the better performance to the smooth images and hasn't the advantage to the images with a large amount of detail. This method, which uses the vector feature to cluster the similar vectors, can avoid the uncertain question and get the better initial codebook. It can be combined with the GLA to further improve the 
performance of final codebook. Because the initial codebook is naturally ordered, the fast searching method of Jiang et al. [7] can be directly used to speed up the clustering of the GLA iteration based on this initializtion method.

In the $k$-dimensional vector space, a query vector $\boldsymbol{X}=$ $\left(x_{1}, x_{2}, \ldots, x_{k}\right)$ and a codebook $\boldsymbol{Y}=\left\{\boldsymbol{Y}_{j} ; i=1, \ldots, N\right\}$ of size $N$ be given. The $L_{2}$-norm of the $k$-dimensional vector $\boldsymbol{X}$ is denoted as $L_{x} . L_{j}$ is the $L_{2}$-norm of the codeword $\boldsymbol{Y}_{j}$. $L_{x}$ and $L_{j}$ can be expressed as

$$
L_{x}=\|X\|=\sqrt{\sum_{i=1}^{k} x_{i}^{2}} ; \quad L_{j}=\| Y_{j} \mid=\sqrt{\sum_{i=1}^{k} y_{i j}^{2}}
$$

The following property can be derived by using the Cauchy-Schwarz inequality:

$$
\begin{aligned}
D(X, Y) & =\|X-Y\|^{2}=\sum_{i=1}^{k}\left(x_{i}-y_{i}\right)^{2} \\
& =\|X\|^{2}+\left\|Y_{j}\right\|^{2}-2 \sum_{i=1}^{k} x_{i} y_{i j} \\
& \geq\|X\|^{2}+\left\|Y_{j}\right\|^{2}-2\|X\|\left\|Y_{j}\right\|=\left(\|X\|-\left\|Y_{j}\right\|\right)^{2} \\
& =\left(L_{x}-L_{j}\right)^{2}
\end{aligned}
$$

Using inequality (2), Jiang constructs a codeword rejection rule as: Supposed the so far minimum Euclidean distance found in a winner search process for the input vector $\boldsymbol{X}$ is $D_{\min }$. If $\left(L_{j}-L_{x}\right)^{2} \geq D_{\min }$ holds, then $\boldsymbol{Y}_{j}$ can be rejected safely. So actual distance computation for obviously unlikely codewords can be avoided.

In the codebook training iterations, the current matching cell of a training vector is obtained from searching the cell of its previous iteration plus the norm-based neighboring cells of its previous cell. Searching process begins from the cell of previous iteration of a training vector. Using inequality (2), only the partial codewords (cells), which have the adjacent norm to the training vector, need an actual distance computation. So the fast cluster can be realized.

\section{Experimental Results}

To show the performance of the proposed algorithm, the gray image Lena and Pepper with the size of $512 \times 512$ pixels are used in the experiment. The vector dimension is 16 (image blocks of $4 \times 4$ ). The initial codebook is generated by using the random algorithm or the proposed method. Table 1 compares the Peak signal-to-noise ratio (PSNR) of two methods. The PSNR is defined as follows:

$$
P S N R=20 \log _{10} \frac{255^{2}}{M S E}
$$

where MSE is the mean-squared-error between original image and its VQ image.

\begin{tabular}{|c|c|c|c|c|c|c|c|c|}
\hline \multirow{2}{*}{\multicolumn{2}{|c|}{ Image Methods }} & \multirow{2}{*}{$\begin{array}{c}\text { itera- } \\
\text { tions }\end{array}$} & \multicolumn{6}{|c|}{ Codebook size $N$} \\
\hline & & & 32 & 64 & 128 & 256 & 512 & 1024 \\
\hline \multirow{4}{*}{ Lena } & & 0 & 14.05 & 14.07 & 14.07 & 20.11 & 29.01 & 30.80 \\
\hline & & 0 & 26.86 & 27.01 & 27.19 & 27.48 & 27.83 & 28.4 \\
\hline & & 20 & 27.98 & 28.51 & 29.22 & 30.23 & 32.08 & 33.2 \\
\hline & & 20 & 8 & 4 & 30.64 & 31.52 & 32.28 & 32.97 \\
\hline \multirow{4}{*}{ epper } & & 0 & & 9 & 53 & 26.44 & 28.41 & 30. \\
\hline & Pro & 0 & 25.87 & 26.04 & 26.19 & 26.41 & 26.73 & 27.25 \\
\hline & Ran & 20 & 27.62 & 28.62 & 29.39 & 30.35 & 31.66 & 32.67 \\
\hline & Proposed & 20 & 27.89 & 29.00 & 30.09 & 31.00 & 31.68 & 32.26 \\
\hline
\end{tabular}

Note in the Table 1 for the initial codebook of size $N=32,64,128$ and 256, the proposed initial algorithm outperforms the random algorithm. For the initial codebook

\begin{tabular}{|c|c|c|c|c|c|c|c|}
\hline \multirow{2}{*}{ Methods } & \multirow{2}{*}{ Cost } & \multicolumn{6}{|c|}{ Codebook size $N$} \\
\hline & & 32 & 64 & 128 & 256 & 512 & 1024 \\
\hline \multirow{5}{*}{$\begin{array}{c}\text { Full } \\
\text { search }\end{array}$} & CMP & 32 & 64 & 128 & 256 & 512 & 1024 \\
\hline & \pm & 1008 & 2000 & 3984 & 7952 & 15888 & 31760 \\
\hline & $x$ & 512 & 1024 & 2048 & 4096 & 8192 & 16384 \\
\hline & Sqrt & 32 & 64 & 128 & 256 & 512 & 1024 \\
\hline & Distortion & 32 & 64 & 128 & 256 & 512 & 1024 \\
\hline \multirow{5}{*}{$\begin{array}{c}\text { Proposed } \\
\text { (Lena) }\end{array}$} & CMP & 7.78 & 11.63 & 18.41 & 28.82 & 46.48 & 76.28 \\
\hline & \pm & 71.07 & 104.18 & 164.37 & 256.77 & 414.67 & 679.60 \\
\hline & $x$ & 59.09 & 94.03 & 157.56 & 255.08 & 421.75 & 701.37 \\
\hline & Sqrt & 0.052 & 0.054 & 0.058 & 0.065 & 0.077 & 0.100 \\
\hline & Distortion & 3.90 & 5.87 & 9.30 & 13.25 & 23.48 & 38.61 \\
\hline \multirow{5}{*}{$\begin{array}{c}\text { Proposed } \\
\text { (Pepper) }\end{array}$} & CMP & 7.21 & 10.64 & 16.57 & 25.69 & 41.47 & 68.53 \\
\hline & \pm & 64.86 & 94.58 & 147.06 & 226.51 & 366.30 & 608.57 \\
\hline & $x$ & 52.53 & 83.89 & 139.28 & 223.13 & 370.67 & 626.39 \\
\hline & Sqrt & 0.052 & 0.054 & 0.058 & 0.065 & 0.076 & 0.098 \\
\hline & Distortion & 3.90 & 5.87 & 9.30 & 11.55 & 23.48 & 38.61 \\
\hline
\end{tabular}

Table 1 Comparison of the PSNR of 0 or 20 iterations using two initial methods.

Table 2 Comparison of the average number of distortion calculations and operations per $\cdot$ vector per iteration after 20 iterations.

size $N=512$ and 1024 , the proportion of nontypical vectors increases with the decrease of the number of training vectors in each cell. So the random initial algorithm shows higher PSNR values than the proposed algorithm. This situation can be avoided in the case of $M \gg N$. By using initial codebook of the proposed method, after 20 iterations, the better codebook can be obtained for the proposed method where $N=1024$ is a nontypical situation. Table 2 summarizes the computation complexities of 20 GLA iterations in terms of the average number of distortion calculations and arithmetical operations per vector in each iteration. The method of Jiang et al. [7] is used to speed up the GLA iteration based on the proposed initializtion method. It shows that the proposed method provides considerable improvement compared to the full search method. In Fig. 1 the proposed method is compared with the random method for codebook size $N=256$ and 20 iterations on "Lena". It shows that the PSNR is improved by $1.3 \mathrm{db}$ using the proposed method.

\section{Conclusion}

This Letter presents an initializtion method of VQ. By using 


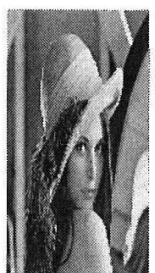

(a)

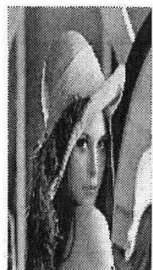

(b)

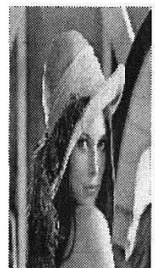

(c)
Fig. 1 Comparison of the result of 20 iterations using the random method and the proposed method for codebook size of 256 on "Lena". (a) Original image, (b) the random method, PSNR $=30.23 \mathrm{db}$, (c) the proposed method, PSNR $=31.52 \mathrm{db}$

vector norm, a robust initial codebook is constructed. After 20 GLA iterations, the average PSNRs improvements of the proposed method are $0.36 \mathrm{db}, 0.71 \mathrm{db}, 1.06 \mathrm{db}, 0.98 \mathrm{db}$, $0.11 \mathrm{db}$ and $0.33 \mathrm{db}$ for the codebook size of 32 to 1024 . The average improvements of the number of " $x$ ", " \pm ", and "CMP" (comparison) operations are 93.1\%, 96.1\%, and $86.5 \%$ for all codebook size, respectively. The average improvement of the number of distortion calculations is $93 \%$ for all codebook size. The computation complexities can further decrease with an increase in the codebook size and the iteration number. The theory and experiment have demonstrated that the proposed method can improve and speed up the GLA. The proposed method can be efficiently applied to smooth images. To images with a large amount of detail, this algorithm is not recommended to be applied.

\section{Acknowledgments}

This work is partially supported by the National Natural Science Foundation of China (No.60602057) and the NSAF Foundation (No.10776040) of National Natural Science Foundation of China, China.

\section{References}

[1] Y. Linde, A. Buzo, and R.M. Gray, "An algorithm for vector quantizer design," IEEE Trans. Commun., vol.28, no.1, pp.84-95, 1980.

[2] A. Laha, N.R. Pal, and B. Chanda, "Design of vector quantizer för image compression using self-organizing feature map and surface fitting," IEEE Trans. Image Process., vol.13, no.10, pp.1291-1303, 2004.

[3] H. Sun, K.-Y. Lam, S.-L. Chung, W. Dong, M. Gu, and J. Sun, "Efficient vector quantization using genetic algorithm," Neural Comput. Applic., vol.14, pp.203-211, 2005.

[4] K.-B. Kim, S. Kim, and G.-H. Kim, "Vector quantizer of medical image using wavelet transform and enhanced SOM algorithm," Neural Comput. Applic., vol.15, pp.245-251, 2006.

[5] K. Thangavel and D. Ashok Kumar, "Optimization of code book in vector quantization,” Ann. Oper. Res., vol.143, pp.317-325, 2006.

[6] S.-E. Qian, "Fast vector quantization algorithms based on nearest partition set search," IEEE Trans. Image Process., vol.15, no.8, pp.24222430, 2006.

[7] S. Jiang, Z. Lu, and Q. Wang, "Fast norm-ordered nearest-neighbor codeword search algorithms for image vector quantization," Chinese J. Electronics, vol.12, no.3, pp.374-376, July 2003. 